\title{
Laser heater commissioning at an externally seeded free-electron laser
}

\author{
S. Spampinati, ${ }^{1,2, *}$ E. Allaria, ${ }^{1}$ L. Badano, ${ }^{1}$ S. Bassanese,${ }^{1}$ S. Biedron, ${ }^{1, \|}$ D. Castronovo, ${ }^{1}$ \\ P. Craievich, ${ }^{1, \dagger}$ M. B. Danailov, ${ }^{1}$ A. Demidovich, ${ }^{1}$ G. De Ninno, ${ }^{1,2}$ S. Di Mitri, ${ }^{1}$ B. Diviacco, ${ }^{1}$ \\ M. Dal Forno, ${ }^{1,3,}$ E. Ferrari, ${ }^{1,3}$ W. M. Fawley, ${ }^{1}$ L. Fröhlich, ${ }^{1,8}$ G. Gaio, ${ }^{1}$ L. Giannessi, ${ }^{1,4}$ \\ G. Penco, ${ }^{1}$ C. Serpico, ${ }^{1}$ C. Spezzani, ${ }^{1, \pi}$ M. Trovò,${ }^{1}$ M. Veronese,${ }^{1}$ \\ S. V. Milton, ${ }^{1, \|}$ and M. Svandrlik ${ }^{1}$ \\ ${ }^{1}$ Elettra Sincrotrone Trieste, 34149 Basovizza (TS), Italy \\ ${ }^{2}$ Laboratory of Quantum Optics, Nova Gorica University, Vipavska 13, Rožna Dolina, \\ SI-5000 Nova Gorica, Slovenia \\ ${ }^{3}$ University of Trieste, 34128 Trieste, Italy \\ ${ }^{4}$ Theory Group-ENEA C.R. Frascati, 00044 Frascati (Rome), Italy
}

(Received 27 September 2014; published 18 December 2014)

\begin{abstract}
FERMI is the first user facility based upon an externally seeded free-electron laser (FEL) and was designed to deliver high quality, transversely and longitudinally coherent radiation pulses in the extreme ultraviolet and soft x-ray spectral regimes. The FERMI linear accelerator includes a laser heater to control the longitudinal microbunching instability, which otherwise is expected to degrade the quality of the high brightness electron beam sufficiently to reduce the FEL output intensity and spectral brightness. In this paper, we present the results of the FERMI laser heater commissioning. For the first time, we show that optimizing the electron beam heating at an upstream location (beam energy, $100 \mathrm{MeV}$ ) leads to a reduction of the incoherent energy spread at the linac exit (beam energy, $1.2 \mathrm{GeV}$ ). We also discuss some of the positive effects of such heating upon the emission of coherent optical transition radiation and the FEL output intensity.
\end{abstract}

DOI: 10.1103/PhysRevSTAB.17.120705

PACS numbers: 29.27.Bd, 41.60.Cr

\section{INTRODUCTION}

The photon pulse generated by an externally seeded freeelectron laser (FEL) inherits the spectral quality of the seed laser and in principle can be nearly fully longitudinally coherent. However, this coherence can be spoiled by several collective processes occurring during the preparation of the electron beam before its entrance to the FEL undulator. Among them, the longitudinal microbunching instability (MBI) is one of the most significant [1,2]. Driven by a combination of longitudinal space charge forces and

\footnotetext{
*Corresponding author.

simone.spampinati@cockcroft.ac.uk

Present address: University of Liverpool, Department of Physics, Liverpool, UK, and Cockroft Institute, Sci-Tech Daresbury, Warrington, UK.

Present address: Paul Scherrer Institute, 5232 Villigen-PSI AG, Switzerland.

${ }^{\ddagger}$ Present address: SLAC National Accelerator Laboratory, Menlo Park, California 94025, USA.

${ }^{\S}$ Present address: Deutsches Elektronen-Synchrotron DESY, 22603 Hamburg, Germany.

"Present address: Laboratoiré de Physique des Solides, UMR8502, Univerité Paris sud 11, Orsay, France.

"Present address: Colorado State University, Fort Collins, Colorado, USA.

Published by the American Physical Society under the terms of the Creative Commons Attribution 3.0 License. Further distribution of this work must maintain attribution to the author(s) and the published article's title, journal citation, and DOI.
}

chromatic dispersion, the MBI induces a broadband modulation of the electron beam energy and longitudinal charge distribution. This process originates with shot noise and cathode-induced nonuniformities in the particle distribution and is further stimulated and amplified by any downstream energy or charge density modulation, e.g., space charge forces in the low energy injector region, nonzero longitudinal impedance in the $\mathrm{rf}$ accelerator cells and drift sections, and the strong chromatic dispersion sections associated with magnetic bunch length compressors that increase the electron bunch peak current to the level required for photon production. Coherent synchrotron radiation (CSR) in these bunch compressors can further enhance the MBI-induced energy and density modulations $[3,4]$. At the undulator entrance, the electron beam can have significant longitudinal energy and density modulations $[1,5,6]$ on the multimicron to submicron scale lengths. The longer wavelength modulations can degrade the FEL spectrum, especially for a seeded source, while those at the shortest wavelengths effectively appear as an increased "slice" energy spread that can reduce the FEL gain and output radiation intensity.

As a means to control the MBI in high brightness linacs, Saldin et al. [7] proposed the addition of a particular device commonly referred to as a "laser heater" (LH). This device adds a controlled amount of incoherent energy spread to the electron beam and suppresses further MBI growth via energy Landau damping [5]. The capability of a LH to increase the final electron beam brightness was initially 
demonstrated at the LCLS hard X-ray FEL, where both a reduction of the FEL gain length and an increase of the photon flux were observed [8]. A reduction of coherent optical transition radiation (COTR) from diagnostic screens was observed and reported as a direct evidence of microbunching suppression at optical frequencies [8-10].

A similar LH system has been installed in the linac driving the FELs of the FERMI user facility [11] at a beam energy of approximately $100 \mathrm{MeV}$ [12]. In this paper we expand upon preliminary results reported in $[10,13]$ and present the performance of the FERMI LH system in terms of both MBI suppression and improved FEL performance. We show that optimized LH heating reduces the compressed electron beam energy modulations and, for the first time, this optimization reduces the incoherent energy spread at the linac exit. More recent results obtained at the LCLS with the new X-band transverse deflecting cavity rf deflection system are in good agreement with our findings $[9,14]$. We also comment upon the LH system's positive effects upon the intensity and the spectral quality of the output FEL pulses.

\section{LINAC LAYOUT AND LASER HEATER SETUP}

The FERMI user facilities are based upon two externally seeded FELs and cover the extreme ultraviolet (FEL-1) [15] and soft X-ray (FEL-2) [16] wavelength ranges. Both FEL lines are driven by a high brightness electron beam produced by the same S-band radiofrequency linear accelerator (rf linac). Due to concerns raised during the initial design of FERMI regarding uncontrolled MBI growth, a LH system was installed at the end of the FERMI injector, at a beam energy of approximately $100 \mathrm{MeV}$ [12]. Figure 1 shows the FERMI accelerator layout. Electrons are generated in a photoinjector [17] that includes the electron gun and two booster accelerating structures. The first linac section (L1) accelerates the beam to $\sim 285 \mathrm{MeV}$ and is followed by the first (BC1) of two bunch compressors employing horizontally deflecting magnetic chicanes. FERMI may run with either just $\mathrm{BC} 1$ or with both $\mathrm{BC} 1$ and the second bunch compressor (BC2) energized. Previous analytical and numerical studies [18-20] suggested that one-stage compression (i.e., BC1 only) minimizes the total MBI gain, resulting in the smallest slice energy spread at the linac. L1 also includes an X-band cavity to linearize the compression process [21-23]. $\mathrm{BC} 2$ is placed five accelerating structures (comprising L2 and L3) after $\mathrm{BC} 1$ at a location corresponding to a beam energy of $\sim 800 \mathrm{MeV}$. A final set of five structures (L4) accelerates the beam to its final energy with a tunable operational range from $\sim 1.0$ to $1.5 \mathrm{GeV}$.

The FERMI LH [10-12] is located in an acceleration-free line upstream of L1. Its electron beam diagnostic suite includes various intercepting screens together with a horizontally deflecting spectrometer. A similar diagnostic layout is installed after $\mathrm{BC} 1$, which includes a vertical rf deflecting cavity [24] to study the time-sliced beam parameters after the longitudinal compression. In combination with a horizontal spectrometer, this deflector allows time-resolved characterization of the beam's longitudinal phase space [25]. A third diagnostic beam line (labeled DBD in Fig. 1) placed at the end of the linac is equipped with both a horizontal and a vertical rf deflecting cavity [26] and is followed by a horizontally deflecting spectrometer.

The FERMI LH consists of a short, planar undulator located in a magnetic chicane where an external infrared laser pulse is superimposed temporally and spatially over the electron beam. The electrons-laser interaction

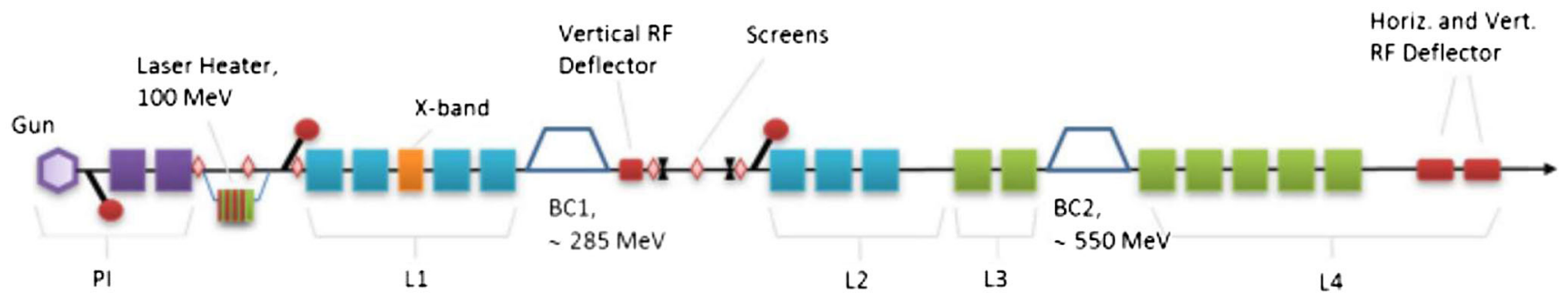

FEL1

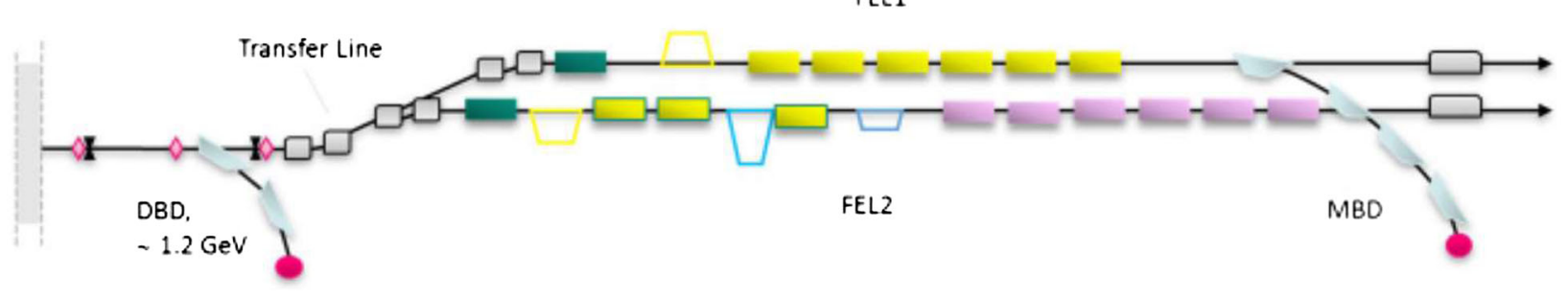

FIG. 1. Sketch of the FERMI S-band accelerator and FEL lines (not to scale). 


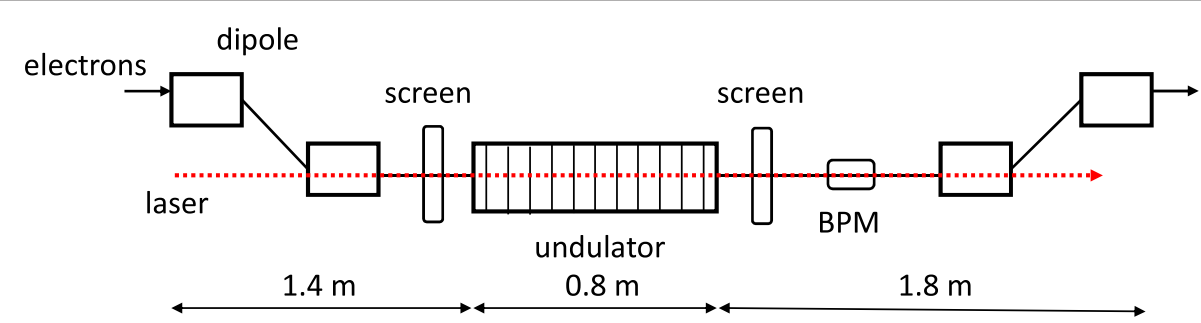

FIG. 2. Schematic of the electron beam trajectory in the laser heater chicane. The first pair of dipole magnets deflects the beam to a line parallel to the linac axis at a distance of $0.03 \mathrm{~m}$ from it, coinciding with the undulator axis. Then the beam is deflected again back to the linac axis by the second pair of magnets.

within the undulator produces an energy modulation on a longitudinal scale length corresponding to the laser wavelength $(783 \mathrm{~nm})$. The second half of the chicane smears the energy modulation in time, leaving the beam with a larger incoherent energy spread than at the LH entrance. Figure 2 illustrates the trajectory of the electron beam in FERMI's LH. Table I lists the main FERMI LH parameters.

The external laser pulse for the FERMI LH consists of a small portion of the overall photocathode Ti:Sa drive laser pulse, removed just before the latter is harmonically upconverted to the UV. The LH pulse is transported to an optical table placed in the linac tunnel near the electron beam vacuum pipe. There, its temporal duration can be adjusted within the range $8-15$ ps by using a grating stretcher. An energy per pulse of up to $160 \mu \mathrm{J}$ is available at the LH undulator, corresponding to a maximum peak power of $20 \mathrm{MW}$. According to theory [5], such power can produce up to $100 \mathrm{keV}$ of energy spread (rms) upon the electron beam. The nominal value of beam heating required by FEL operation with optimal MBI suppression is expected to be around $10 \mathrm{keV}$ (rms) [19]. The LH laser pulse energy can be attenuated by a polarimeter down to a minimum value of approximately $0.25 \mu \mathrm{J}$. A separate shutter can completely block the pulse and in the following text this situation is referred to as "LH off". The initial phase of the LH commissioning was carried out at full laser power to detect more clearly the induced heating.

The undulator gap can be remotely changed to resonantly match the external laser wavelength for electron beam energies in the range $95-120 \mathrm{MeV}$. The relative energy bandwidth of the undulator is $4 \%$, while the

TABLE I. FERMI laser heater parameters.

\begin{tabular}{lcc}
\hline \hline Parameter & Value & Units \\
\hline Undulator period & 40 & $\mathrm{Mm}$ \\
Number of periods & 12 & $\ldots$ \\
Undulator parameter $K$ & $0.8-1.17$ & $\ldots$ \\
Laser wavelength & 783 & $\mathrm{~nm}$ \\
Laser pulse duration, FWHM & $8-15$ & $\mathrm{ps}$ \\
Laser energy & $<160$ & $\mu \mathrm{J}$ \\
Laser transverse size (at waist), rms & $130-220$ & $\mu \mathrm{m}$ \\
Horizontal offset in the chicane & 30 & $\mathrm{~mm}$ \\
Chicane bending angle & 3.5 & Degree \\
\hline \hline
\end{tabular}

gap-undulator strength parameter calibration has an error of the order of $0.3 \%$. The nearby horizontal spectrometer (see Fig. 1) allows measurement of the average electron beam energy to better than $1 \%$ relative error.

The LH chicane includes two scintillating multiscreen stations equipped with Chromox (Al2O3:Cr) targets, one on each side of the undulator (see Fig. 2). The Chromox screens image both the laser and the electron beam to help ensure their mutual spatial overlap. Three remotely controllable steering mirrors, one in the laser room and two on the optical table at the chicane's entrance, permit adjustment of the laser's centroid position relative to that of the electron beam. The steering mirrors on the laser table are mounted on a piezo tip-tilt stage, allowing control of the transverse position to better than $10 \mu \mathrm{m}$ accuracy. Based on these mirrors together with the image of a "virtual undulator focus" imaged on-line on a CCD, a laser beam position feedback system for cancellation of the slow spatial drifts has been recently implemented.

Figure 3 shows the laser transverse profile and the electron beam measured at the two screens at either end of the undulator. The laser beam comes to $\mathrm{a} \approx 220 \mu \mathrm{m}$-sized waist at the center of the undulator in both transverse planes, while the electron beam size is $\approx 130 \mu \mathrm{m}$, also in both transverse planes (all rms values). Since both the

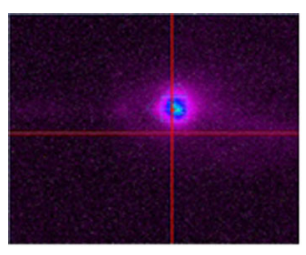

(a)

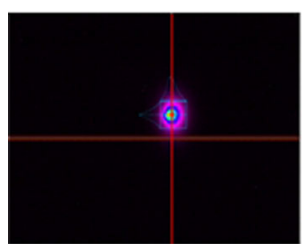

(c)

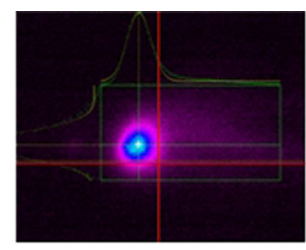

(b)

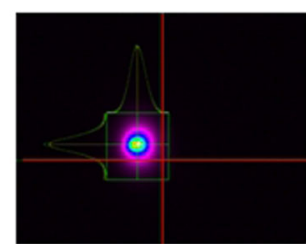

(d)
FIG. 3. Image of the LH laser [(a), (b)] and electron beam [(c), (d)] on two Chromox screen targets at the undulator entrance $[(a),(c)]$ and exit $[(b),(d)]$. The red crosses indicate the center of the screen. 
electron beam and the external LH laser originate with the photocathode infrared drive laser, they are naturally locked in time. The temporal overlap between the electron bunch and the infrared laser pulse is adjusted empirically to an accuracy of a few femtoseconds by scanning a laser delay line while observing the electron beam heating. Recent results suggest that solely relying upon minimization of COTR emission downstream of $\mathrm{BC} 1$ to set the timing may bias the center toward a high current "spike" (typically appearing in the beam head during high compression situations). This may lead to nonoptimum FEL performance.

\section{MEASUREMENT OF ELECTRON BEAM HEATING}

\section{A. Longitudinal phase space}

When the electron and the laser beams are superimposed both in space and in time, the laser-electron interaction in the undulator generates significant energy spread that is detectable in the downstream $\mathrm{BC} 1$ spectrometer line (see Fig. 1). Here the beam is stretched vertically with an rf deflector [24] before entering the spectrometer. This deflection maps the electron-beam longitudinal coordinate to the vertical coordinate on a cerium-doped yttrium aluminum garnet (YAG) intercepting screen after the spectrometer dipole magnet; the dipole converts the particles energies to the screen horizontal coordinate. The magnetic transport lattice [27] produces a horizontal energy dispersion $\eta_{x} \approx 0.58 \mathrm{~m}$ [28] and a horizontal betatron function $\beta \approx 1 \mathrm{~m}$ at the YAG screen location. Hence, for a normalized emittance of $\sim 1 \mu \mathrm{m}$, it is possible to reconstruct the beam longitudinal phase space and to measure the slice energy spread along the longitudinal bunch coordinate [25], as shown in Fig. 4.

Figure 4 shows the longitudinal phase space measured when the LH was off (a) and when the beam was fully heated (b). The energy profiles of a small temporal portion of the beam bounded between the black lines, corresponding to no heating and full heating, are reported in Figs. 4(c) and 4(d), respectively. For these measurements the $\mathrm{rf}$ acceleration of the L1 section was phased to be on crest (otherwise the resultant energy chirp would be overwhelmingly dominant). We measured a minimum slice energy spread of $40 \mathrm{keV}$ rms with the LH turned off. However, this value is significantly larger than the one expected just out of the injector $\left(\sigma_{E} \sim 5 \mathrm{keV}\right)$. In fact, the spectrometer measurement system resolution is limited by the combination of beam transport optics-related energy resolution, the minimum diagnostic screen resolution, and the rf deflectorinduced slice energy spread [29]. Moreover, the slice energy spread includes a contribution from the residual rf curvature, i.e., the projection of the $\mathrm{z}$-E correlation into the slice one. In agreement with the predictions of the theoretical model of Ref. [5], the LH-heated beam shows a characteristic "double-horn" energy distribution [Fig. 4(d)] (a)

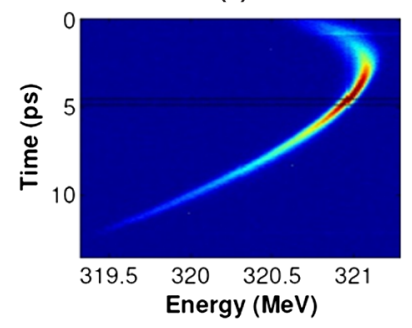

(c)

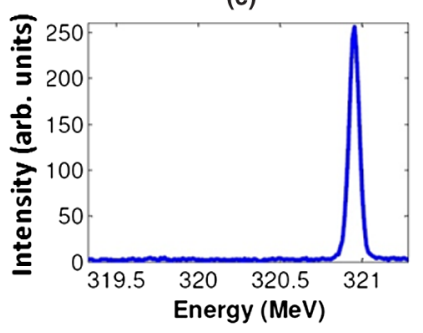

(b)

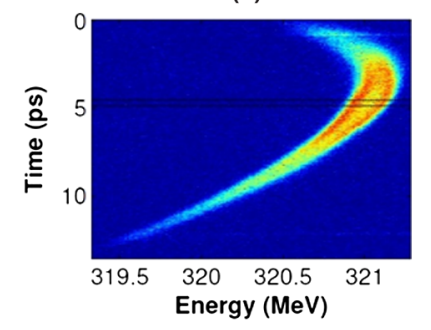

(d)

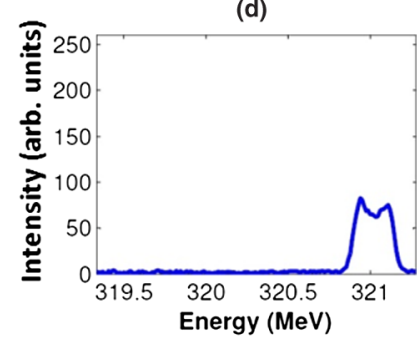

FIG. 4. Electron beam longitudinal phase space reconstructed from measurements at the $\mathrm{BC} 1$ spectrometer line. (a) Laser heater off. (b) Laser heater on with $160 \mu \mathrm{J}$ energy per pulse. (c) Energy profiles of a central portion of the beam delimited by the black lines in Fig. 4(a) when the heater is off. (d) Energy profiles of a central portion of the beam delimited by the black lines in Fig. 4(b) when the heater is on.

as expected when the laser transverse profile is significantly larger than that of the electron beam. In contrast, when the laser size matches the electron beam transverse size the energy distribution approaches a Gaussian profile (not shown here). This latter configuration has been predicted theoretically to be more effective in suppressing the MBI [5]. Nevertheless, during the LH commissioning, whose results are discussed in the remainder of this section and also in Sec. IVA (with the exception of the last measurement of Sec. IV A reported in Fig. 12), we used a relatively large laser spot (a waist size of $\approx 220 \mu \mathrm{m} \mathrm{rms} \mathrm{in} \mathrm{both}$ transverse planes) to simplify the electron and the laser beam spatial overlap procedure. The laser pulse has a time duration of 13 ps in these measurements.

\section{B. Heating dependence upon undulator gap}

Figure 5 depicts the measured energy spread of a central beam time slice (hereafter referred to as "slice energy spread") vs the undulator gap size. During these measurements the LH laser energy was fixed to $160 \mu \mathrm{J}$. The slice energy spread is computed from the second moment of a fitting function $[17,30]$ built upon a "super-Gaussian" function described in [31]. The experimental points are obtained by subtracting in quadrature the energy spread measured with LH off from the value measured with LH on. Doing so, one ideally obtains the energy spread added by the LH only, presuming they add in quadrature. The error bars are from the statistical measurement error over 15 shots and from estimated errors on the beam mean energy and energy dispersion ( $1 \%$ and 5\%, respectively). 


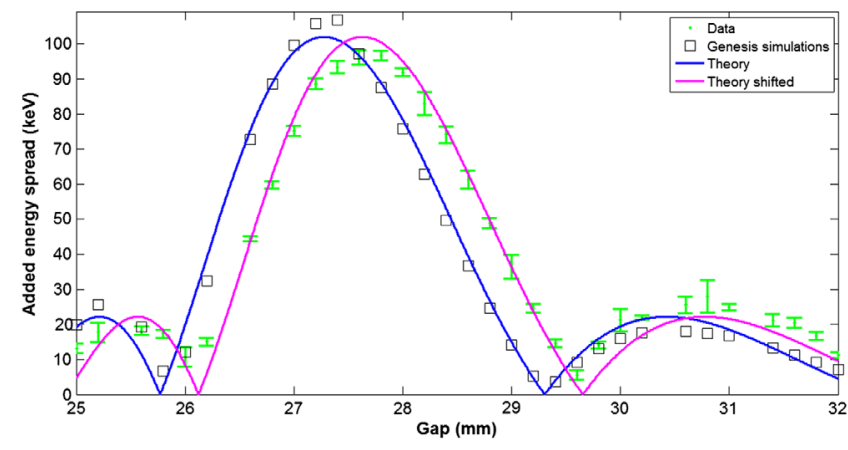

FIG. 5. Electron beam slice energy spread added by the LH vs undulator gap: theoretical (solid lines) and experimental (green dots). The black squares are simulation results from the GENESIS1.3 simulation code.

The black square points reproduce results obtained with the simulation code GENESIS1.3 [32]. The blue curve (solid, peak on the left) depicts the theoretical behavior of the energy spread as a function of the normalized undulator strength parameter $K$ [see Eq. (2)]. This is in turn given by the gap calibration curve that is proportional to the sinc function of the detuning between the laser wavelength and the undulator resonance wavelength:

$$
\sigma_{\varepsilon}(g)=\sigma_{\varepsilon} \max \left|\frac{\sin \left\{\frac{\pi N\left[\lambda(g)-\lambda_{L}\right]}{\lambda_{L}}\right\}}{\frac{\pi N\left[\lambda(g)-\lambda_{L}\right]}{\lambda_{L}}}\right|,
$$

where we define

$$
\lambda(g)=\left[\frac{\lambda_{u}}{2 \gamma_{0}^{2}}\left(1+\frac{K(g)^{2}}{2}+\frac{\varepsilon_{N, x} \gamma_{0}}{\beta_{x}}+\frac{\varepsilon_{N, y} \gamma_{0}}{\beta_{y}}\right)\right] .
$$

Here $\gamma_{0}$ is the electron beam mean relativistic Lorentz factor, $g$ is the undulator gap, $\varepsilon_{N, x}$ and $\varepsilon_{N, y}$ are the electron beam transverse normalized rms emittance in the two transverse planes and $\beta_{x}$ and $\beta_{y}$ are the average betatron functions across the undulator (the beam optics are symmetric in the two transverse planes along the undulator). Equation (2) is valid for both the laser and the electron beams propagating along the undulator axis without any tilt angle. The relative contribution of the emittance terms to the resonance wavelength is approximately $0.01 \%$. The magenta line in Fig. 5 is obtained shifting the blue curve the equivalent of $-0.5 \mathrm{MeV}$ in beam energy to match better the experimental data points. This shift is quantitatively compatible with aforementioned expected accuracies of the undulator strength calibrations and energy spectrometer measurements.

\section{Slice energy spread}

After the optimum tuning of the undulator gap was achieved experimentally, the evolution of the slice energy spread as a function of the laser energy was recorded (see Fig. 6) with excellent agreement between experiment and theory [8] as given by

$$
\sigma_{\varepsilon}=\frac{K \cdot J J \cdot m_{e} c^{2}}{\gamma_{0}} \sqrt{\frac{P_{L}}{P_{0}}} \int_{0}^{L_{U}} \sqrt{\frac{\sigma_{L}^{2}(z)}{\sigma_{e}^{2}+\sigma_{L}^{2}(z)}} \frac{1}{\sigma_{L}(z)} d z
$$

Here $z$ is the longitudinal coordinate along the undulator, $\sigma_{e}$ and $\sigma_{L}$ are, respectively, the rms transverse sizes of the electron and of the laser beam, $P_{L}$ is the laser beam peak power, $J J$ is the normal Bessel function difference undulator coupling factor [33] and $P_{0}=8.9 \mathrm{GW}$. Equation (3) was originally derived in Ref. [8], in which it was demonstrated to accurately reproduce LCLS experimental data. However, here we modify it slightly to take into account the laser diffraction along the undulator. The transverse size of the electron beam is approximately constant along the undulator. Close inspection of Fig. 6 shows very good agreement between theory and measurement (within the experimental error bars) for heating below $50 \mu \mathrm{J}$ and a small $(\sim 5 \mathrm{keV})$ difference at higher values.

Figure 7 shows the behavior of the slice energy spread along the electron bunch with the LH off and the LH run at the maximum laser energy of $160 \mu \mathrm{J}$. Here the magenta-colored curve represents the empirically calculated LH-induced energy spread only, obtained by quadratic subtraction of the LH-off green-colored curve from the LH-on red-colored curve. The slice energy spread measured without the LH is dominated by the rf curvature at the bunch edges, and by the optics resolution plus the deflector contribution in the bunch core. All these contributions are equally present when the heater is on and are then removed by the quadratic subtraction performed to obtain the magenta-colored curve. Beam heating varies within $20 \%$ of the $80-\mathrm{keV}$ mean value along the bunch. The bluecolored line shows the added slice energy spread as predicted by the ELEGANT code [34] presuming an intrinsic energy spread of $5 \mathrm{keV}$. The measured and predicted curves are in good agreement. Note that for this large level of

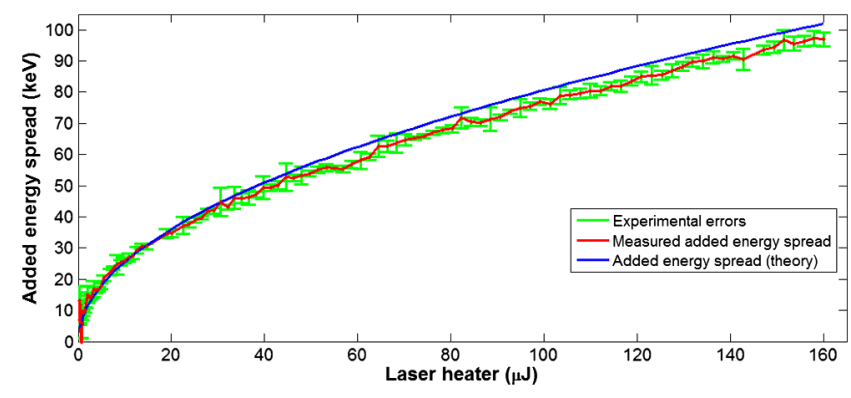

FIG. 6. Laser heater induced rms slice energy spread, theoretical (solid line) and experimental (dots), as a function of the laser energy per pulse. 


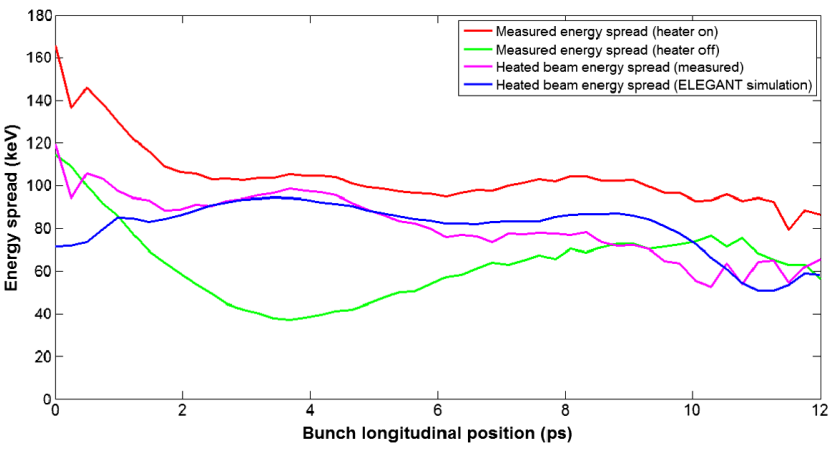

FIG. 7. Slice energy spread along the electron bunch. The green curve shows the energy spread measured with the LH off while the red curve shows the energy spread of the beam when heated at the maximum LH laser energy of $160 \mu \mathrm{J}$. The magenta curve is obtained by subtracting in quadrature the LH-off from the LH-on curve. The blue curve shows the slice energy spread as predicted by the ELEGANT code.

heating, the contribution of the intrinsic energy spread is quite small.

The temporal variation of the heating along the electron beam arises from a number of effects including variation of electron beam optics $[11,17]$ and a nonconstant transverse laser-electron alignment caused by the coupling of the LH chicane dispersion $\eta_{x}$ with the electron beam energy curvature [17].

\section{SUPPRESSION OF THE MICROBUNCHING INSTABILITY}

\section{A. One-stage compression (BC1 only)}

In this section we analyze the effects of the LH heating on the electron beam dynamics downstream of $\mathrm{BC} 1$, for a configuration of one active bunch compressor only. One observed a clear evidence of MBI effects when the LH was off and when the compression factor (CF) exceeded 3. Data shown in this section were acquired for a $2.8 \mathrm{ps}(\mathrm{rms})$ long, $500 \mathrm{pC}$ charge electron beam that is shortened by about a $\mathrm{CF} 5.5$ in $\mathrm{BC} 1\left(\mathrm{R}_{56}=-41 \mathrm{~mm}\right)$. The X-band cavity installed upstream of $\mathrm{BC} 1$ was switched off during these measurements so that the magnetic compression acted upon a nonlinear energy profile and produced a ramped current distribution close to that shown in [35], with a peak current $\sim 350 \mathrm{~A}$. In this case, both the current and the energy spectrum of the beam appeared temporally modulated at the end of the linac. This is shown in Fig. 8(a) where the energy profile was imaged on the screen of the spectrometer line in the diagnostic beam dump (labeled DBD in Fig. 1). The high frequency modulation is attributed to the MBI while the longer time-scale structures likely arise from nonlinearities in the compression process. In Fig. 8(b), the LH laser energy was approximately $0.4 \mu \mathrm{J}$, equivalent to a heating of $5 \mathrm{keV}(\mathrm{rms})$ for a spot size of $220 \times 220 \mu \mathrm{m}^{2}$ (rms) and the pulse duration of $\sim 13$ ps (FWHM). Here the

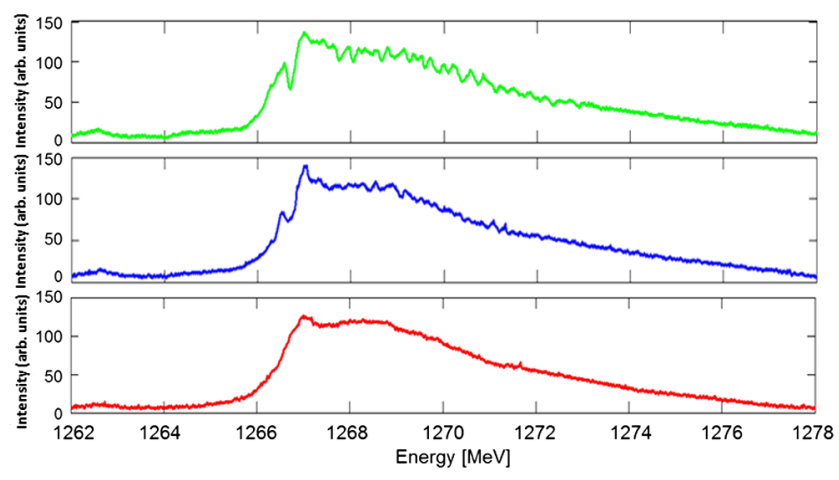

FIG. 8. 500 pC, 350 A peak current electron beam energy profile determined at the diagnostic beam dump (DBD) spectrometer, at the end of the linac, for three levels of LH beam heating: no heating (top), $5 \mathrm{keV}$ rms (middle), $15 \mathrm{keV}$ rms (bottom). These heating values are calculated from the measured values of the laser parameters according to Eq. (3).

high frequency structures apparent in Fig. 8(a) are much reduced in amplitude but the longer time-scale current structures (e.g., the feature at $1267 \mathrm{MeV}$ ) remain almost unchanged by this small level of heating. When the laser energy was increased to $\approx 3 \mu \mathrm{J}$ (equivalent to $15 \mathrm{keV}$ beam heating) or greater, even the longer scale structures are nearly smeared out and the high frequency structures are essentially eliminated [Fig. 8(c)].

Another indication of strong MBI modulation of the FERMI electron beam was the presence of coherent optical transition radiation (COTR) [36,37] produced when the beam passed through OTR screens, as previously reported [38]. Figure 9 shows the integrated OTR signal from a screen placed after the transfer line in front of the FEL-1 undulator (see the lower panel of Fig. 1), as a function of the energy spread added by the LH. Here the data were taken for a $350 \mathrm{pC}$ beam charge, a $\mathrm{CF} \sim 10$ produced by $\mathrm{BC} 1$ only. The $\mathrm{X}$-band linearizer was activated to produce a flat longitudinal current profile at the accelerator exit.

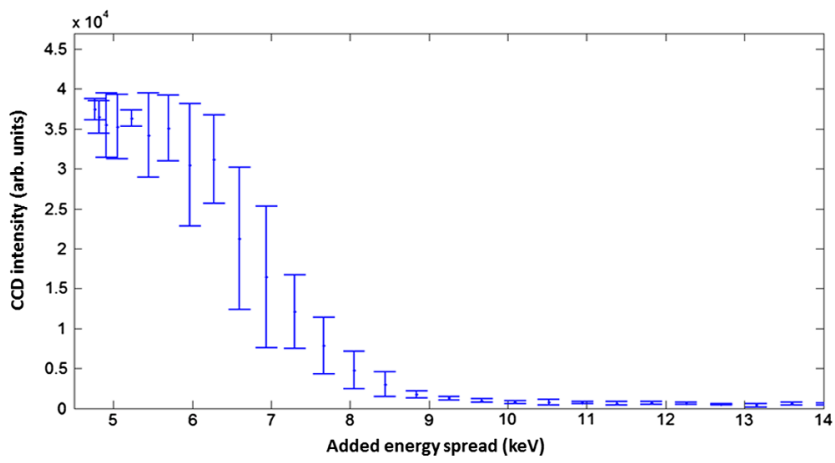

FIG. 9. OTR intensity measured at linac exit plotted vs laser heater-added energy spread, for a $350 \mathrm{pC}$ bunch charge compressed by a factor 10 in BC1. During these measurements and those in Fig. 10, the LH laser spot size was $220 \times 220 \mu \mathrm{m}^{2}(\mathrm{rms})$ at its waist and the pulse duration was $\sim 13$ ps (FWHM). 
The OTR integrated intensity decayed with increasing LH heating and approached a constant level when the energy spread added by the LH exceeded $\approx 8 \mathrm{keV}$ (corresponding to the laser energy of $0.7 \mu \mathrm{J}$ ). This value of constant intensity was equal to the incoherent level obtained by inserting an upstream OTR screen on the beam path. More precisely, the addition of an uncorrelated energy spread to the electron beam larger than $8 \mathrm{keV}$ removed any beam modulation observable by the CCD camera detector over its sensitivity range $0.2 \mu \mathrm{m}<\lambda<0.9 \mu \mathrm{m}$. A similar result was obtained for a beam charge of $500 \mathrm{pC}$ when examining the OTR signal at the first diagnostic screen downstream of $\mathrm{BC} 1$ (see Fig. 1). In this case, the OTR intensity approached an asymptotic level for laser energies above $1 \mu \mathrm{J}$ (equivalent to $10 \mathrm{keV}$ of added energy spread), as shown in Fig. 10.

This LH-induced suppression of microbunching-caused COTR in the linac allowed diagnostic use of OTR screens (with improved spatial resolution relative to the YAG screens) for measurement of the transverse and the longitudinal particle distribution. Figure 11 shows the transverse beam spot imaged on the OTR screen BC01.03 with the LH off and on. This screen is located downstream of $\mathrm{BC} 1$ and, importantly, is used to measure the transverse emittance of the beam immediately following compression in the chicane. Without LH heating, the apparent beam spot is contaminated by COTR and significantly fluctuates from shot to shot which makes emittance measurements highly

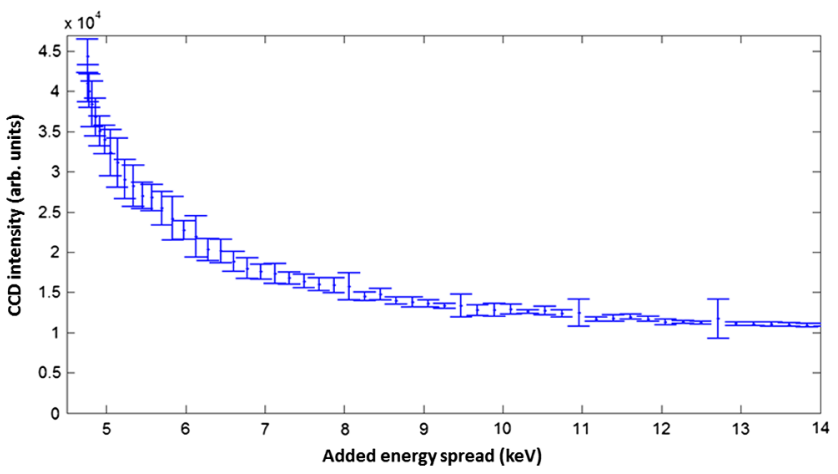

FIG. 10. OTR intensity measured immediately downstream of $\mathrm{BC} 1$ plotted vs laser heater-added energy spread for a $500 \mathrm{pC}$ bunch charge compressed by a factor 10 in $\mathrm{BC} 1$.
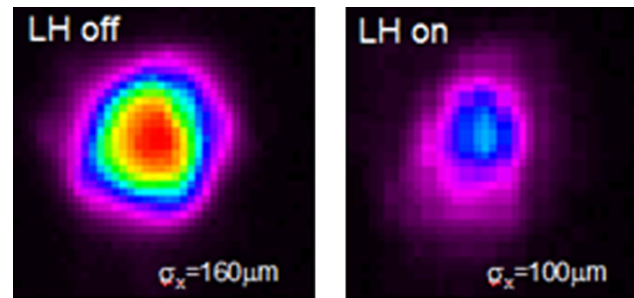

FIG. 11. Transverse electron beam profile imaged on the screen BC01.03 downstream of BC1, with LH off (left) and on (right).

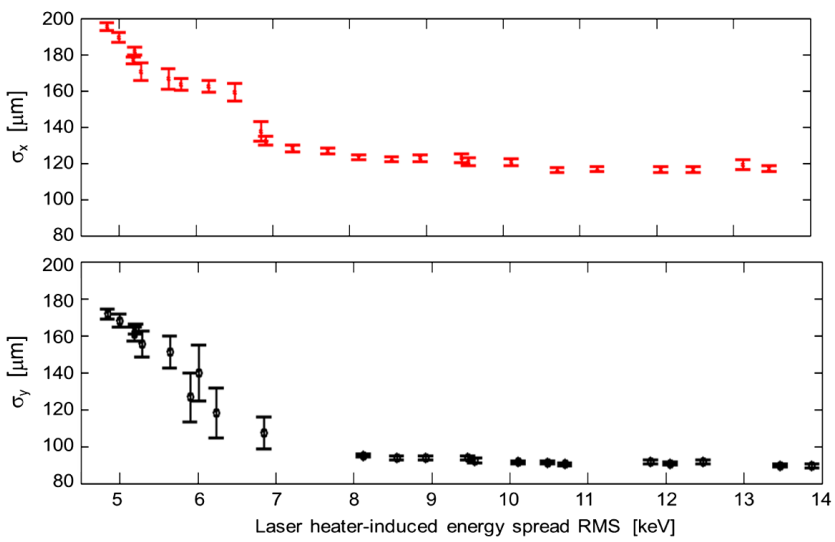

FIG. 12. Measured transverse electron beam dimensions in $X$ and $Y$ at the linac exit as a function of the LH heating strength. For these particular measurements the laser spot size was $130 \times$ $130 \mu \mathrm{m}^{2}$ (rms) and the pulse duration was $\sim 15$ ps (FWHM).

unreliable. With the LH on at a high heating value, the measured transverse sizes are both considerably smaller and also essentially constant on a shot-to-shot basis.

Figure 12 shows the transverse dimensions of the beam at the linac exit as a function of the laser heating. The measurements were made at the last OTR screen placed just before the transfer line to the FELs. For low heating the beam image is contaminated by COTR, increasing the measured beam sizes. The measured dimensions decrease as the heating is increased and reach almost constant values for heating values greater than $8 \mathrm{keV}$.

\section{B. Two-stage compression (both $\mathrm{BC} 1 \&$ BC2 activated)}

At various times in 2012 and 2013 we have operated FERMI employing active bunch length compression from both $\mathrm{BC} 1$ and $\mathrm{BC} 2$ compressors. With this double compressor setup we have found clear evidence that appropriate setting of the FERMI LH intensity results in a final incoherent energy spread at the linac exit smaller than that corresponding to no LH use at all.

Figure 13 shows the time-resolved electron beam longitudinal phase space measured at the diagnostic beam dump (DBD) spectrometer for three different settings of the LH. Figure 14 shows the energy profiles determined from the central portion of the beam, delimited by the black lines in Fig. 13, for three different LH settings corresponding to heating values (before compression) of 4.5, 10.5, and $29.5 \mathrm{keV}$ as determined according to Eq. (3) using the measured values of $130 \times 130 \mu \mathrm{m}^{2}$ (rms) spot size and $\sim 15$ ps (FWHM) pulse duration. One sees from Fig. 14 that the middle heating value corresponds to a smallest final energy spread. Also note the laser beam size now matches well that of the electron beam and the energy profiles no longer have hornlike features.

Figure 15 shows the calculated slice energy spread based upon measurements at the end of the linac, as a function of 
(a)

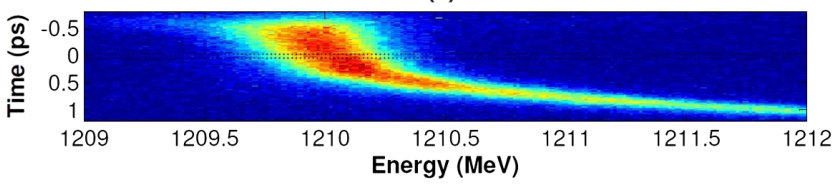

(b)

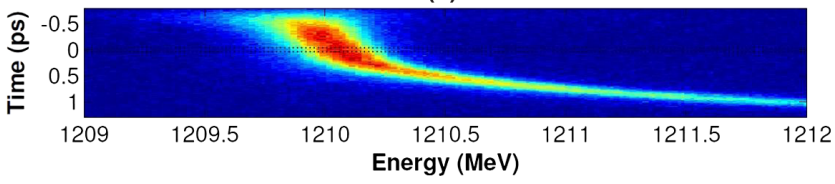

(c)

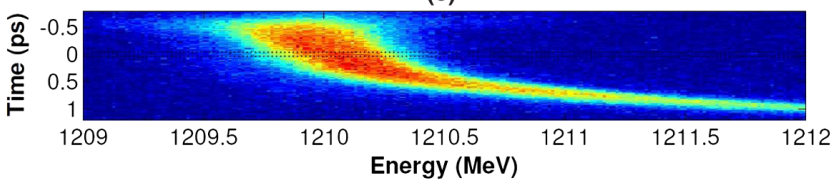

FIG. 13. Beam longitudinal phase space at the end of the linac for three settings of the laser heater resulting in calculated heating values (before bunch length compression) of $4.5 \mathrm{keV}$ (top panel), $10.5 \mathrm{keV}$ (middle) and $29.5 \mathrm{keV}$ (bottom) (rms). These are uncompressed values, relevant upstream of $\mathrm{BC} 1$. During these measurements the LH laser spot was reduced to $130 \times 130 \mu \mathrm{m}^{2}(\mathrm{rms})$.

the energy spread added by the LH in this two-stage compression configuration. Here the electron beam charge was $500 \mathrm{pC}$ and the total compression factor $(\mathrm{CF})$ of $\sim 6.5$ was approximately evenly split between $\mathrm{BC} 1$ and $\mathrm{BC} 2$ resulting in a final peak current of $\sim 400 \mathrm{~A}$. The LH heating ranged from 5 to $85 \mathrm{keV}$ before compression and was determined according to Eq. (4) using the measured laser parameters mentioned in the previous paragraph. As discussed previously, the measured uncorrelated energy spread will be larger than the true value because of measurement effects such as energy spread added by the rf deflector system, smearing associated with spectrometer

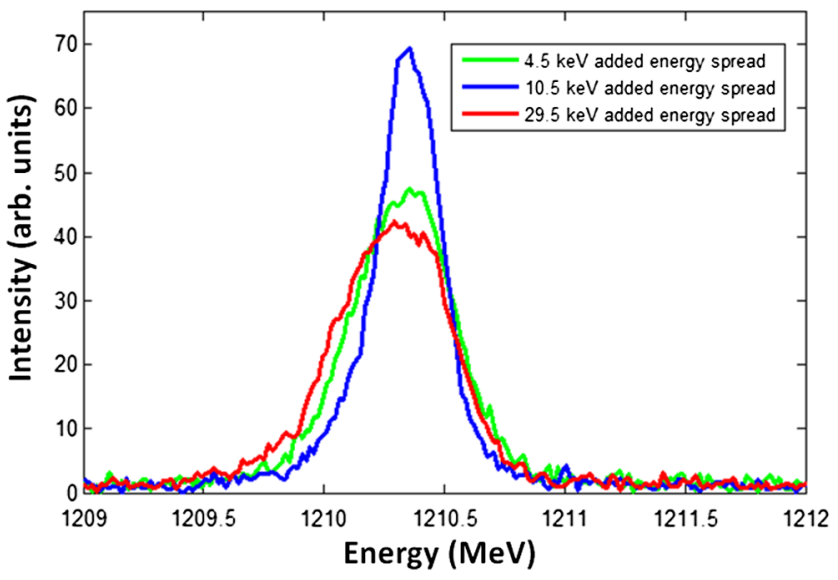

FIG. 14. Energy profiles of a central portion of the beam delimited by the black lines in Fig. 13 for three settings of the laser heater. Note that the measured profile no longer has the hornlike features, indicating a very similar transverse spot size for the laser and the electron beam.

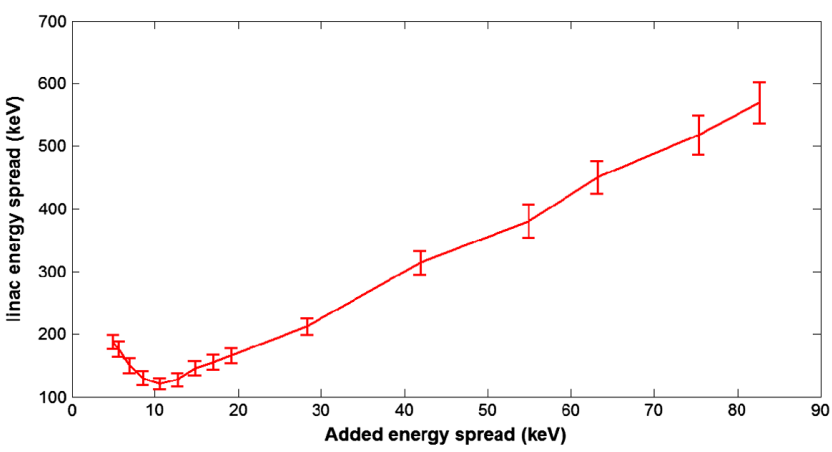

FIG. 15. Slice energy spread as a function of the energy spread added by the $\mathrm{LH}$, for a $500 \mathrm{pC}$ bunch charge compressed in both $\mathrm{BC} 1$ and $\mathrm{BC} 2$ with a total $\mathrm{CF}=7$. Each data point is the average over seven discrete measurements with the plotted error bars calculated as the standard deviation.

beam transport optics, and the limited resolution of the intercepting screen system. At the DBD diagnostic station at the linac exit, we estimate that these effects together lead to a minimum energy spread resolution of approximately $75 \mathrm{keV} \mathrm{rms}$ in this configuration. In order to estimate the true electron beam energy spread, we have subtracted this $75 \mathrm{keV}$ value in quadrature from the measured energy spread. The resultant value is plotted in Fig. 15 and shows that a local minimum of $\sim 120 \mathrm{keV}$ energy spread at linac exit appears for a LH heating level of $10 \mathrm{keV}(\mathrm{rms})$. At smaller heating levels, we measured a slice energy spread at the end of the linac of about $200 \mathrm{keV}$, due to unsuppressed MBI growth. Larger heating also results in a larger final energy spread, due to the downstream (from the LH) beam compression in $\mathrm{BC} 1$ and $\mathrm{BC} 2$ and the approximate preservation of the longitudinal emittance.

\section{LH EFFECTS UPON FEL PERFORMANCE}

FERMI's FEL-1 [15] and FEL-2 [16] are externally seeded, single stage, harmonic upshift FELs based on the principle of high gain harmonic generation [39]. As previously reported $[10,40]$ beneficial effects of increased energy spread from the LH have been observed on the FEL-1 output radiation pulse energy over the full current operating range in wavelength from $65 \mathrm{~nm}$ down to $17 \mathrm{~nm}$. These results are similar to the improvements in the LCLS output pulse energy due to LH heating [8]. A very detailed study concerning the effective extension of the harmonic upshift range for FERMI's FEL-1 due to non-Gaussian energy spread heating effects of the LH was recently presented in Ferrari et al. [40]. That paper (i.e., Figs. 3 and 4) found that optimized heating could more than triple the FEL output over the nonheating situation. Under some specific circumstances in terms of electron beam parameters, LH and FEL parameters, we have also seen that LH action can significantly improve the FEL-1 output spectra [13] in terms of central wavelength stability, spectral shape and bandwidth. We have seen similar 
qualitatively beneficial effects concerning the output of FEL-2 over a wavelength range of 5 to $13 \mathrm{~nm}$. However, as with FEL-1, the quantitative improvements depend upon multiple system factors (e.g., harmonic upshift ratios in the two stages, current waveform, final output wavelength, etc.) and we will defer a highly detailed discussion of these phenomena to a future paper. Nonetheless, it is fair to write that essentially in all cases some LH heating leads to improvements compared to no heating at all.

\section{CONCLUSIONS}

We have presented details concerning the configuration and performance of the FERMI laser heater (LH) system. The measured heating of the electron beam is in excellent agreement with analytical predictions. Measurements both of the electron beam longitudinal phase space and of the COTR intensity produced at intercepting screens indicate that sufficient heating by the LH system strongly suppresses the overall longitudinal microbunching instability (MBI) growth in several linac settings with different compression schemes (one and two compressors) in a weak to mild ( $\mathrm{CF} \sim 5-10)$ compression regime. These results are in agreement with earlier LH studies at LCLS [8], which operated in a much stronger (CF 90) compression regime. The optimal heating level for suppression of the instability is in the range $7-10 \mathrm{keV} \mathrm{rms}$ as measured at the LH location. Depending upon the details of the beam pulse compression scheme (i.e., use of only one vs two compression sections) and the total compression factor, we have demonstrated for the first time that a LH can reduce the slice energy spread at accelerator exit, presumably due to the suppression of the MBI. As was previously found at the LCLS [8], optimized tuning of the LH heating improves the FEL radiation output energy. In at least some FERMI parameter regimes, there is also evidence of a positive impact of the LH upon FEL spectral purity and stability.

\section{ACKNOWLEDGMENTS}

This work was funded by the FERMI project of ElettraSincrotrone Trieste, partially supported by the Ministry of University and Research under Grants No. FIRBRBAP045JF2 and No. FIRB-RBAP06AWK3.

[1] E. L. Saldin, E. A. Schneidmiller, and M. V. Yurkov, Nucl. Instrum. Methods Phys. Res., Sect. A 490, 1 (2002).

[2] T. Shaftan and Z. Huang, Phys. Rev. ST Accel. Beams 7, 080702 (2004).

[3] T. Limberg, P. Piot, and E. A. Schneidmiller, Nucl. Instrum. Methods Phys. Res., Sect. A 475, 353 (2001).

[4] S. Heifets, S. Stupakov, and S. Krinsky, Phys. Rev. ST Accel. Beams 5, 064401 (2002).
[5] Z. Huang, M. Borland, P. Emma, J. Wu, C. Limborg, G. Stupakov, and J. Welch, Phys. Rev. ST Accel. Beams 7, 074401 (2004).

[6] S. Di Mitri et al., Nucl. Instrum. Methods Phys. Res., Sect. A 608, 19 (2009).

[7] E. L. Saldin, E. A. Schneidmiller, and M. V. Yurkov, Nucl. Instrum. Methods Phys. Res., Sect. A 528, 355 (2004).

[8] Z. Huang et al., Phys. Rev. ST Accel. Beams 13, 020703 (2010).

[9] D. Ratner (private communication).

[10] S. Spampinati et al., in Proceedings of the 34th Free Electron Laser Conference, (JACoW, Nara, Japan, 2012), MOPD58.

[11] C. J. Bocchetta et al., Report No. ST/F-TN-07/12, 2007 [https://www.elettra.trieste.it/FERMI/index.php?n=Main .CDRdocument].

[12] S. Spampinati, S. Di Mitri, and B. Diviacco, in Proceedings of the 29th Free Electron Laser Conference, Novosibirsk, Russia (BINP, Novosibirsk, 2007), WEPPH015.

[13] S. Spampinati et al., in Proceedings of the 35th International Free Electron Laser Conference (JACoW, New York, NY, 2013), WEPS067.

[14] J. Welch, in Proceedings of the 32nd International Free Electron Laser Conference, FROAli, Malmö, Sweden (Max-lab, Sweden, 2010).See also A. H. Lumpkin, ICFA Newsletter 63, 16 (2014).

[15] E. Allaria et al., Nat. Photonics 6, 699 (2012).

[16] E. Allaria et al., Nat. Photonics 7, 913 (2013).

[17] G. Penco et al., JINST 8, P05015 (2013).

[18] M. Cornacchia et al., LBNL Technical Note No. 62765, 2007 [https://publications.lbl.gov/islandora/object/ir\% 3A128747].

[19] M. Venturini, Phys. Rev. ST Accel. Beams 10, 104401 (2007).

[20] S. Di Mitri, M. Cornacchia, S. Spampinati, and S. Milton, Phys. Rev. ST Accel. Beams 13, 010702 (2010).

[21] T. Smith, in Proceedings of the 1984 Linear Accelerator Conference (Stanford University, Stanford, CA, 1986), p. 421; also SLAC Report No. 303, 1986.

[22] P. Emma, SLAC Report No. LCLS-TN-01-1, 2001.

[23] K. Flöttmann, T. Limber, and Ph. Piot, Report No. TESLAFEL-2001-06, 2001.

[24] P. Craievich et al., in Proceedings of the 32nd International Free Electron Laser Conference, Malmö, Sweden (Max-lab, Sweden, 2010), WEPB43.

[25] G. Penco et al., in Proceedings of the 34th International Free Electron Laser Conference (JACoW, Nara, Japan, 2012), WEPD20.

[26] M. Dal Forno et al., in Proceedings of the 4th International Particle Accelerator Conference, IPAC-2013, Shanghai, China, 2013 (JACoW, Shanghai, China, 2013), THPFI010.

[27] S. Di Mitri and M. Cornacchia, in Proceedings of the 32nd International Free Electron Laser Conference, Malmö, Sweden (Max-lab, Sweden, 2010), WEPB42.

[28] S. Di Mitri, L. Fröhlich, and G. Penco, Report No. ST/FTN-12/04, 2012.

[29] C. Behrens, Z. Huang, and X. Dao, Phys. Rev. ST Accel. Beams 15, 022802 (2012).

[30] S. Di Mitri, E. M. Allaria, P. Craievich, W. Fawley, L. Giannessi, A. Lutman, G. Penco, S. Spampinati, and M. Trovo, Phys. Rev. ST Accel. Beams 15, 020701 (2012). 
[31] F.-J. Decker, SLAC Report No. SLAC-PUB 6684, 1994.

[32] S. Reiche, Nucl. Instrum. Methods Phys. Res., Sect. A 429, 243 (1999).

[33] W. B. Colson, IEEE J. Quantum Electron. 17, 1417 (1981).

[34] M. Borland, ANL/APS Report No. LS-287, 2000.

[35] E. Allaria et al., Phys. Rev. ST Accel. Beams 17, 010704 (2014).

[36] V. L. Ginzburg and V. N. Tsytovich, Transition Radiation and Transition Scattering (Adam Hilger, Bristol, UK, 1990).
[37] D. W. Rule and R. B. Fiorito, AIP Conf. Proc. 229, 315 (1991).

[38] H. Loos et al., in Proceedings of the 30st International Free Electron Laser Conference (JACoW, Geongju, Korea, 2008), THBAUP01.

[39] L. H. Yu, Phys. Rev. A 44, 5178 (1991).

[40] E. Ferrari, E. Allaria, W. Fawley, L. Giannessi, Z. Huang, G. Penco, and S. Spampinati, Phys. Rev. Lett. 112, 114802 (2014). 